\title{
基于双目视觉的电缆护套断裂伸长率自动测量
}

\author{
张格悠 ${ }^{1)}$ ，龚俊 ${ }^{2}$ ，陈俊松 ${ }^{1)}$ ，张志东 ${ }^{3)}$ ，朱策 ${ }^{4)}$, 刘凯 ${ }^{1)^{*}}$ \\ ${ }^{1)}$ (四川大学电气工程学院 成都 610065) \\ 2) (成都产品质量检验研究院有限责任公司 成都 610199) \\ 3) (中国石油集团川庆钻探工程有限公司安全环保质量监督检测研究院 成都 610056) \\ 4) (电子科技大学信息与通信工程学院 成都 611731) \\ (kailiu@scu.edu.cn)
}

\begin{abstract}
摘 要: 针对电缆护套断裂伸长率的人工测量方法精度差、自动程度低的缺陷, 提出一种基于双目视觉的自动测量 方法. 首先采集试样拉伸视频，对首帧图像采用基于信息熵的算法定位 2 个圆环标记符圆心坐标，若正确识别则使用 圆心坐标作为标记点, 否则选取立体匹配结果良好的 SURF 特征点中距离圆心最近的点作为标记点; 然后用金字塔 L-K 光流法追踪隔帧采样后的护套拉伸视频中的标记点, 基于计算出的标记点三维坐标和加速度判定试样断裂的粗 略帧位置, 再回溯 2 个采样间隔进行逐帧检测, 最终精确定位断裂帧并计算断裂伸长率．在电缆护套质检现场进行实 验的结果表明，以 240 帧/ $\mathrm{s}$ 高速相机的测量结果作为真值，采用所提方法测得断裂伸长率的测量均方根误差为 $7.13 \%$, 低于人工测量方法的 $21.84 \%$ ，处理速度达到了 107.8 帧/s，在实际应用中能明显提升检测的精度、速度和自动化程度.
\end{abstract}

关键词：双目视觉；三维重建；断裂伸长率；加速鲁棒特征；金字塔 L-K 光流

中图法分类号: TP391.41 DOI: 10.3724/SP.J.1089.2021.18779

\section{Automatic Measurement of Elongation at Break of Cable Sheath Based on Binocular Vision}

\author{
Zhang Geyou $^{1)}$, Gong Jun ${ }^{2)}$, Chen Junsong ${ }^{1)}$, Zhang Zhidong ${ }^{3)}$, Zhu Ce ${ }^{4)}$, and Liu Kai ${ }^{1)^{*}}$ \\ 1) (College of Electrical Engineering, Sichuan University, Chengdu 610065) \\ 2) (Chengdu Institute of Product Quality Inspection Co., Ltd., Chengdu 610199) \\ 3) (CNPC Chuanqing Drilling Engineering Company Limited, Chengdu 610056) \\ 4) (School of Information and Communication Engineering, University of Electronic Science and Technology of China, Chengdu 611731)
}

\begin{abstract}
In order to solve the problem of accuracy and automation of manual measurement for elongation at break of cable sheath, an automatic measurement based on binocular vision is proposed. Firstly, after the sample tensile video is collected, location method based on information entropy is used on the first frame for the center coordinates of two circle markers, if markers are correctly identified, the center coordinate is used as the marked point, otherwise the point closest to the center of circle in SURF feature points with good stereo matching results is selected as the marked point. Secondly, the marked points tracking based on L-K optical flow is used in the sampled video of sheath stretching process, the determination of the rough frame position of sheath breaking is based on the calculated three-dimensional coordinates and acceleration, then two sampling intervals are backtracked for frame by frame detection. Finally, the breaking frame is located

收稿日期：2020-11-15; 修回日期：2021-07-20. 基金项目：国家自然科学基金面上项目(61473198); 四川省科技厅重点研发项目 (2020YFG0029). 张格悠(1998一), 男, 硕士研究生, 主要研究方向为双目视觉、结构光三维成像及应用; 龚俊(1970一), 男, 学士, 正 高级工程师, 主要研究方向为质检信息化和智能化、机器视觉、数据分析和管理等; 陈俊松(1996一), 男, 硕士研究生, 主要研究方向 为图像识别、目标追踪; 张志东(1974一), 男, 博士, 高级工程师, 硕士生导师, 主要研究方向为油气装备故障诊断、在线监测、数据 分析等; 朱策(1969-), 男, 博士, 教授, 博士生导师, 主要研究方向为视频编码与通信、视频分析与处理等; 刘凯(1973一), 男, 博士, 教授, 博士生导师, 论文通讯作者, 主要研究方向为结构光三维成像及应用、机器视觉、数字图像/信号处理等.
\end{abstract}


precisely then elongation at break is calculated. The experimental results in the field of quality inspection of cable sheath show that the measured root mean square error of elongation at break is $7.13 \%$ which is lower than that of manual measurement method (21.84\%), compared with the true values measured by 240 fps high-speed camera, and the processing speed of the method reaches $107.8 \mathrm{fps}$. Propose method can obviously improve the detection accuracy, speed and automation in practical application.

Key words: binocular vision; 3D reconstruction; elongation at break; speeded-up robust features; pyramidal L-K optical flow

电缆护套材料的断裂伸长率是衡量电缆质量 的重要参数, 根据 GB/T 2951.11-2008 标准, 其定 义是：试件拉伸至断裂时, 标记距离的增量与未拉 伸试样的标记距离的百分比. 断裂伸长率反映出 材料在拉应力下的承受能力, 在韧性材料中间接 地反映材料的拉伸性能. 材料的拉伸性能是结构 强度设计的主要依据之一, 可以为预测材料的其 他力学性能提供参考, 如疲劳、耐刮擦和磨损等. 目前质检机构采用机械量具进行人工检测，该方 法使用拉力机拉伸电缆护套, 在与护套平行处放 置一把测量尺，同时测量人员通过目测读取断裂 瞬间的护套长度. 该方法需要工作人员长时间操 作测量设备, 人工录人断裂伸长率数据, 人工成本 高, 精度低, 效率低下且有一定的危险性. 因此, 如何能精确、自动地测定电缆护套的最大拉伸量是 一个具有充分研究价值的问题.

目前尚无基于立体视觉技术测量断裂伸长率 的方法. 双目视觉技术具有非接触、设备简单和适 用于动态目标等优点, 因此, 本文基于双目视觉技 术对电缆护套上的标记点进行三维测量. 该技术 被广泛应用于视觉定位、自动避障和尺寸测量等方 面. 陈浩等 ${ }^{[1]}$ 提出了一种应用于裸眼立体显示系统 的双目跟踪算法, 比传统方法提升了跟踪精度和 稳定性, 适用于医学等高精度领域. 解则晓等 ${ }^{[2]}$ 设 计的双目测量系统在零件圆孔轮廓的尺寸测量中 达到很高的精度. 崇爱新等 ${ }^{[3]}$ 提出链式双目相机三 维测距算法, 通过精确三维重建实现高精度的无 缝线路钢轨纵向位移测量.

光流原理常被应用于目标追踪中，可用于对 标记点的准确跟踪. 光流 ${ }^{[4]}$ 的概念 1950 年被首次 提出, 光流场的计算 ${ }^{[5]} 1981$ 年被提出, 而后由 Lucas 和 Kanade 提出了改进光流算法. 最终, Bouguet $^{[6]}$ 提出了金字塔 L-K 光流法. 在工程应用 中, 张德嘉等 ${ }^{[7]}$ 基于增强 PyrLK 光流法解决了传统 光流法不支持特征点旋转和双向匹配的问题, 实现
了树木骨架模型的三维重建; 齐乃新等 ${ }^{[8]}$ 提出的多 尺度分层跟踪视觉里程计算法, 用 L-K 光流法对 前后帧图像特征点进行了亚像素精度的数据关联, 使定位精度达到了厘米级; 唐钰欣等 ${ }^{[9]}$ 提出了一 种利用光流场原理测量物体面形的方法, 其基于 Brox 光流估计 2 帧图像间观测点的位移, 即可精 确地恢复物体的高度信息.

本文提出的基于双目视觉的电缆护套断裂伸 长率测量方法, 通过双目相机采集视频, 根据双目 图像求解三维空间中的标记点坐标和间距, 自动 检测断裂帧的最大拉伸长度并计算输出断裂伸长 率, 实现了自动的三维测量和计算.

\section{1 本文检测系统和算法流程}

本文的测量系统由一台双目相机、一台计算 机、一台拉力仪、一个光源和一套支撑装置组成, 被测样本置于微机控制电子万能试验机(俗称拉力 仪)的夹具上, 整个测量系统实物如图 1 所示, 预 先完成双目标定后, 即可由程序控制视频采集和 处理.

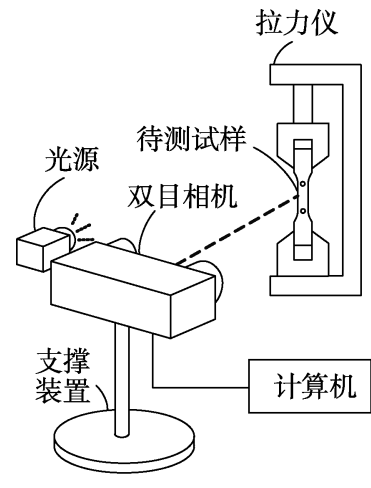

a. 测量装置原理简图

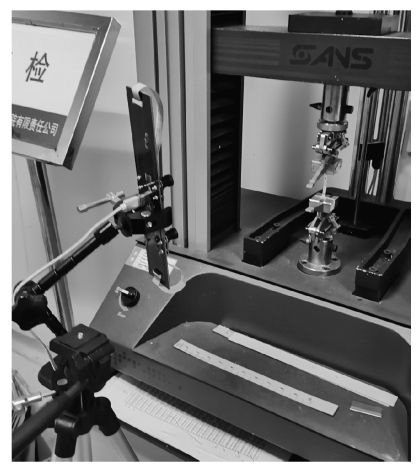

b. 系统实物图
图 1 本文测量系统

算法 1. 电缆护套断裂伸长率自动测量算法.

Step1. 在试样上标记特征点, 启动拉力机拉伸试 样直至断裂, 采集整个过程的双目视频. 
Step2. 通过基于信息熵的圆心提取算法进行特征 提取并用 SURF 算法进行立体匹配，定位 2 个标记点， 计算初始三维间距.

Step3. 以 Step2 中提取的标记点为初始坐标，隔帧 采样视频, 对标记点进行跟踪, 并实时计算存储 2 个标 记点的三维坐标和加速度.

Step4. 当加速度与均值偏离超过 5 倍标准差且大 于某预设常数时判定试样断裂，将参数回溯到至少 2 个 采样帧前开始逐帧检测，直至再次检测到断裂帧，将该 帧前一帧的标记点间距作为断裂间距，计算断裂伸长率.

\section{2 标记点的定位}

不同种类的电缆护套试样颜色不同但均匀. 本文采用直径为 $0.4 \mathrm{~cm}$ 的圆环标记点, 用印章在 试样上进行标志，如图 2 所示. 将自动识别、定位 标记点用于后续进行光流跟踪, 标记点的识别只 在首帧中进行.

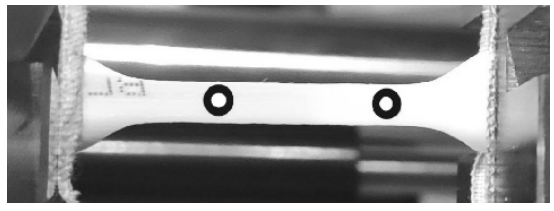

图 2 被测试样上的标记符

\section{1 标记符圆心定位}

先对图像用最小类间方差法求取图像二值化 阈值 $T_{\mathrm{b}}$, 分别以 $0.5 T_{\mathrm{b}}$ 和 $1.5 T_{\mathrm{b}}$ 作为 Canny算法的低 阈值、高阈值进行边缘检测，并剔除过大或过小的 连通域后，标记符的边缘轮廓就在其中.

圆形的标记符投影在相机成像平面上呈椭圆 形状，因此计算所有连通域的圆形度

$$
R_{0}=\frac{4 \pi S}{l^{2}}
$$

其中, $S$ 为连通域的实际面积; $l$ 为连通域的周长. 本文选取 $R_{0}>0.7$ 的连通域作为可疑标记符连通域.

图像一维灰度信息熵描述了图像中存在多少 随机性(或不确定性), 即图像的信息量是多少 ${ }^{[10]}$. 其计算方式为

$$
E=-\sum_{j=0}^{255} p(j) \ln [p(j)]
$$

其中, $p(j)$ 为灰度值为 $j$ 的像素点在图像中的出 现概率. 圆环标记符之间的矩形区域图像的灰度 分布集中, 如图 3 所示. 因此, 这片区域内的像素 灰度直方图呈现如图 4 所示的单峰分布, 由于小部 分灰度值占据了很高的出现概率，因此该区域灰度
的不确定性很低，而其他任意 2 个可疑标记符之间 的图像灰度分布相对均匀，不确定性高.

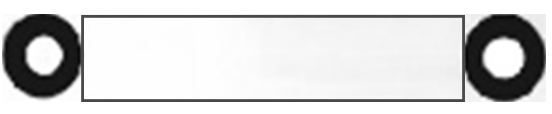

图 3 标记符中间图像(矩形框内)

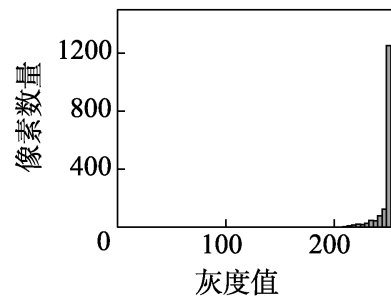

a. 真正标记符中间图像

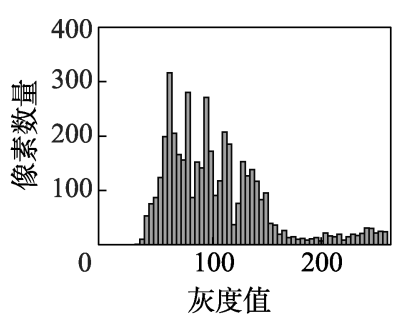

b. 可疑标记符中间图像
图 4 灰度直方图

遍历所有可疑标记点组合，选取中间图像信 息熵最低的一组连通域作为最终提取出的 2 个标 记符连通域, 提取结果如图 5 所示.

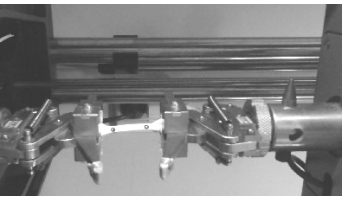

a. 原图

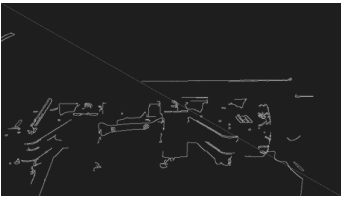

c. 保留适中连通域

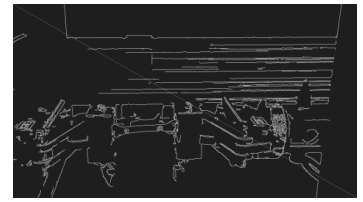

b. 边缘检测

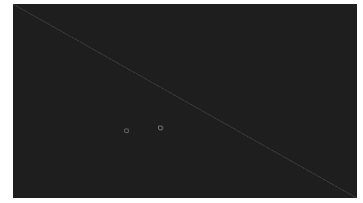

d. 根据圆度和信息熵剔除
图 5 提取标记符

为了准确定位标记连通域的圆心，需要对标 记点的边缘检测结果进行椭圆拟合. 求最小二乘 拟合目标函数，即

$$
\begin{aligned}
& f(A, B, C, D, E)= \\
& \quad \sum_{i=1}^{n}\left(x_{i}^{2}+A x_{i} y_{i}+B y_{i}^{2}+C x_{i}+D y_{i}+E\right)^{2}
\end{aligned}
$$

其中, $A, B, C, D$ 和 $E$ 均为椭圆标准方程中的系数. 将所有椭圆边缘点组成的方程构成矩阵形式

$$
\left[\begin{array}{ccccc}
x_{1} y_{1} & y_{1}^{2} & x_{1} & y_{1} & 1 \\
\vdots & \vdots & \vdots & \vdots & \vdots \\
x_{i} y_{i} & y_{i}^{2} & x_{i} & y_{i} & 1
\end{array}\right]\left[\begin{array}{c}
A \\
B \\
C \\
D \\
E
\end{array}\right]=\left[\begin{array}{c}
-x_{1}^{2} \\
\vdots \\
-x_{i}^{2}
\end{array}\right]
$$

并求最小二乘解, 即可获得椭圆方程系数的值 ${ }^{[11]}$, 进而获取标记符的圆心坐标. 


\subsection{SURF 特征点提取和匹配}

第 2.1 节中已经获取了标记符的圆心坐标, 若 没有发生误识别, 则可直接将标记符圆心作为标 记点坐标, 借助圆心的绝对位置关系完成双目匹 配, 并进行光流跟踪. 但标记符的误识别是无法避 免的，因此，在发生误识别的情况下，需要通过手 动点击确定圆心的大致位置, 再搜索距离此位置 最近的加速鲁棒特征(speeded-up robust features, SURF) 特征点作为跟踪的初始位置, 进而借助 SURF 描述子进行双目匹配. 首先需要提取 2 个标 记符圆心附近的 SURF 特征点进行立体匹配. SURF 在具备良好的仿射和亮度不变性 ${ }^{[12]}$ 的同时, 具有亚像素的精度和低于尺度不变特征变换(scaleinvariant feature transform, SIFT)的计算量 ${ }^{[13-14]}$, 可 保证三维重建达到足够高的精度.

首先构造矩形感兴趣区域，如图 6 所示. 标记 符初始像素间距为 $l_{0}$, 该矩形区域以 2 个标记符圆 心坐标的平均值为中心, 长边方向与标记符连线 方向一致, 边长取 $1.5 l_{0}$. 随后只对该矩形内的图像 进行 SURF 特征点提取，这会减少误匹配，提升匹 配速度。

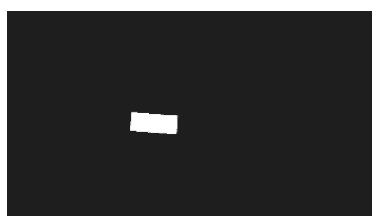

a. 左目

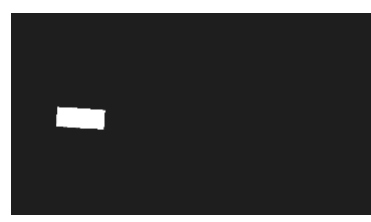

b. 右目
图 6 特征点提取区域掩膜

在感兴趣区域内提取 SURF 特征点的结果如 图 7 所示, 可以看到, 每个圆环标记符的中心位置 都提取出了一个 SURF 特征点.

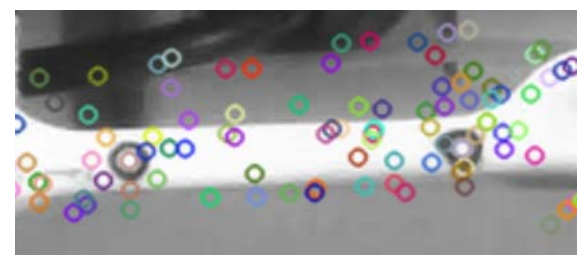

图 7 感兴趣区域特征点提取结果

在进行特征匹配时，由于左右目特征点应满 足极线约束，故应该在各模板的对应的极线两侧 一定宽度内搜索其匹配点, 以齐次形式的右目中 的特征点 $p_{\mathrm{R}}$ 为模板点, $p_{\mathrm{R}}$ 在左相机对应的极线 参数为

$$
\left(\begin{array}{lll}
a_{\mathrm{L}} & b_{\mathrm{L}} & c_{\mathrm{L}}
\end{array}\right)=\boldsymbol{p}_{\mathrm{R}}^{\mathrm{T}} \boldsymbol{F}
$$

其中, $\boldsymbol{F}$ 为基本矩阵, 可通过标定获取的参数进 行计算. 遍历左目特征点 $\boldsymbol{p}_{\mathrm{L}}$ 中与极线的距离 $e_{\mathrm{L}}$ 小 于 4 个像素点, 与模板点 $\boldsymbol{p}_{\mathrm{L}}$ 进行匹配, 获取最佳 匹配点. $e_{\mathrm{L}}$ 计算公式为

$$
e_{\mathrm{L}}=\frac{\mid\left(\begin{array}{lll}
a_{\mathrm{L}} & b_{\mathrm{L}} & c_{\mathrm{L}}
\end{array}\right) \boldsymbol{p}_{\mathrm{L}}}{\mid{ }_{a_{\mathrm{L}}{ }^{2}+b_{\mathrm{L}}^{2}}^{2}}
$$

最后剔除特征向量间欧几里得度量大于 0.3 的 匹配点对. 沿极线搜索的匹配结果几乎没有误匹 配点, 如图 8 所示.
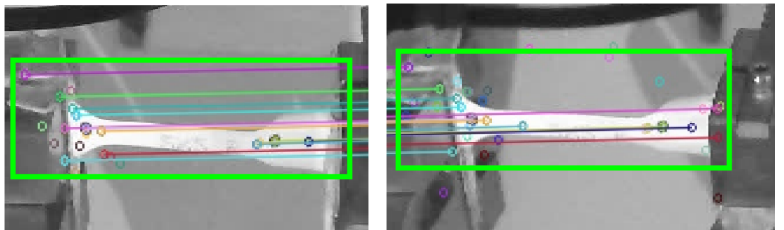

图 8 沿极线搜索的匹配结果

从最终的匹配结果中选取距离 2 个标记符圆 心最近的 SURF 匹配点对, 作为标记点定位结果, 如图 9 所示.

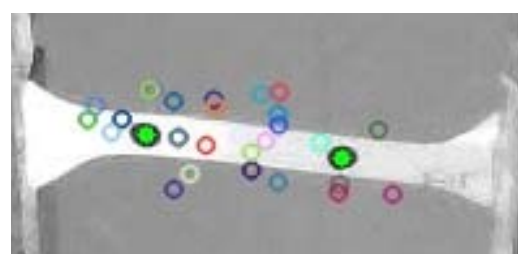

图 9 标记点定位结果(实心圆点)

\section{3 基于金字塔 $\mathbf{L - K}$ 光流法的标记点跟踪}

在提取出标记点后, 还需要持续跟踪其坐标 直到断裂发生, 才能获取试样断裂前瞬间标记点 的三维坐标.

光流是空间运动物体在观察成像平面上的像 素运动的瞬时速度. 光流的计算基于 3 个假设: (1) 亮度一致假设；(2) 时间连续假设；(3) 空间一致假 设. 由于光照基本保持不变, 标记点有一定的面 积，而且运动速度较为缓慢，符合上述 3 个假设.

虽然护套在拉伸过程中会发生形变，但在形 变过程中满足相邻处的速度连续, 因此, 目标点足 够小的邻域内的各个像素运动速度近似相等, 满足 光流计算的空间一致假设, 不会带来额外的误差.

本文采用改进的 L-K 光流法 ${ }^{[15]}$ ，通过引人金 字塔降采样空间, 以改善原方法对目标移动过快 跟踪效果差的缺陷 ${ }^{[16]}$. 考虑一个像素在一帧的 $\mathrm{d} t$ 
时间内, 移动了 $(\mathrm{d} x, \mathrm{~d} y)$ 到下一帧，根据亮度恒定 不变假设, 有

$$
\begin{gathered}
I(x+\mathrm{d} x, y+\mathrm{d} y, t+\mathrm{d} t)-I(x, y, t)= \\
\frac{\partial I}{\partial x} \mathrm{~d} x+\frac{\partial I}{\partial y} \mathrm{~d} y+\frac{\partial I}{\partial t} \mathrm{~d} t+\varepsilon=0
\end{gathered}
$$

则转化为求极小值点的问题. 忽略高阶无穷小量, 并对时间求微分 $\mathrm{d} t$ 得到

$$
\left\{\begin{array}{l}
\frac{\partial I}{\partial x} \frac{\mathrm{d} x}{\mathrm{~d} t}+\frac{\partial I}{\partial y} \frac{\mathrm{d} y}{\mathrm{~d} t}+\frac{\partial I}{\partial t} \frac{\mathrm{d} t}{\mathrm{~d} t}=I_{x} u+I_{y} v+I_{t}=0 \\
I_{t} \mathrm{~d} t=\mathrm{d} I_{x=x_{0}, y=y_{0}}
\end{array}\right.
$$

其中, $I_{x}$ 和 $I_{y}$ 均可用差分计算; $\left(x_{0}, y_{0}\right)$ 为跟踪点的 坐标. 可以用相邻 2 帧同一点的灰度差值计算. 根 据空间一致假设，可以用双线性插值求邻近的 $m \times m$ 个点像素值, 本文取 $m=21$, 求 $u, v$ 最小二乘 解, 即

$$
(u, v)=\operatorname{argmin}[L(u, v)]
$$

其中， $L(u, v)$ 表示最小二乘的平方损失函数，与 $\left(x_{0}, y_{0}\right)$ 邻近的 $m \times m$ 个点相关. 为了在相机发生抖 动或标记点运动速度较快时仍能较为准确地跟踪, 采用基于金字塔的迭代求解 ${ }^{[6]}$, 如图 10 所示. 将 图像逐阶降采样构成金字塔, 计算上层的光流增 量 $d^{(l)}$, 以获取下层的光流初值

$$
g^{(l+1)}=2\left(g^{(l)}+d^{(l)}\right)
$$

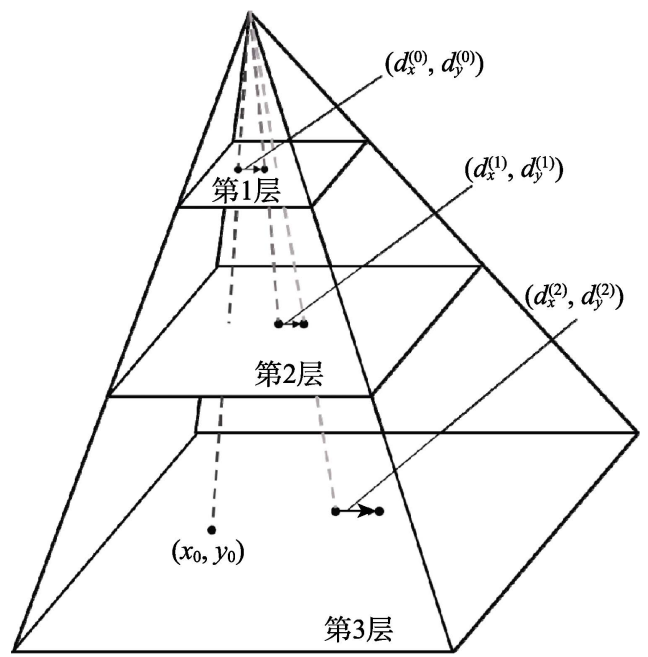

图 10 金字塔 $\mathrm{L}-\mathrm{K}$ 光流法示意图 ${ }^{[6]}$

并在下一层同样用式(9)求取光流增量 $\boldsymbol{d}^{(l)}$, 逐层 迭代至底层，求出最终的光流

$$
\boldsymbol{g}^{(L)}=\sum_{l=0}^{L} 2^{l} \boldsymbol{d}^{(l)}
$$

从而可以计算目标点在下一帧的像素坐标对每帧 的左右目图像的标记点进行光流跟踪，本文中金
字塔层数 $l=3$. 试样拉伸过程中的检测结果如图 11 所示.

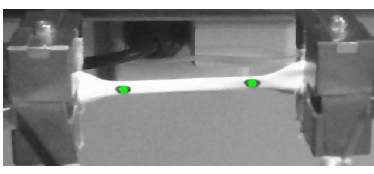

a. 第 200 帧

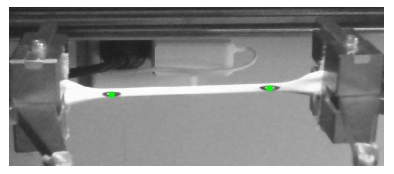

b. 第 400 帧
图 11 L-K 光流法追踪结果(左目)

\section{4 实时三维测量与断裂检测}

\section{1 标记点的实时三维测量}

通过标记点的提取、匹配和光流跟踪，获得了 标记点在左右目中的二维坐标序列, 结合标定好 的内外参数即可基于三角测量的原理解出标记点 的三维坐标序列.

考虑三维空间中的一个标记点, 其三维坐标 为 $\boldsymbol{W}=\left(\begin{array}{llll}X & Y & Z\end{array}\right)^{\mathrm{T}}$. 该点在左右相机成像的像素 坐标通过光流跟踪已知, 分别为 $\left(x_{1}, y_{1}\right),\left(x_{2}, y_{2}\right)$, 从三维坐标投影到二维像素点的过程表示为

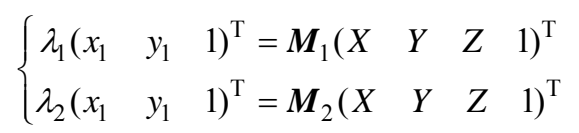

其中, $\boldsymbol{M}_{1}, \boldsymbol{M}_{2}$ 分别为左右相机的 $3 \times 4$ 投影矩阵, 包含了 2 个相机内参矩阵、旋转矩阵和平移向量信 息; $\lambda_{1}, \lambda_{2}$ 为尺度变换因子. 整理式(12), 可得到 有 4 个方程的线性方程组, 用最小二乘法解出标记 点的三维坐标 ${ }^{[17]}$.

根据求得的每帧三维坐标 $\boldsymbol{W}_{t}$ 、差分计算标记 点的第 $t$ 帧的速度 $v_{t}$ 和加速度 $a_{t}$, 即

$$
\left\{\begin{array}{l}
v_{t+1}=\frac{\left(W_{t+1}-W_{t}\right)}{\Delta t} \\
a_{t+1}=\frac{\operatorname{norm}\left(v_{t+1}-v_{t}\right)}{\Delta t}
\end{array}\right.
$$

并计算标记点三维距离 $l_{t}$ 大小. 其中, norm 表示取 $L_{2}$ 范数; $\Delta t$ 为光流追踪时相邻 2 帧的实际时间差.

由于第 0 帧和第 1 帧时无法计算加速度，本文 置 $a_{0}, a_{1}=0$. 如图 12 所示, 如在第 $t+1$ 到第 $t+2$ 帧 之间发生了断裂，那么在第 $t+2$ 帧的处理中，计算 出的速度 $v_{t+2}$ 的模长就会发生突变, 进而导致计 算出的加速度 $a_{t+2}$ 发生突变触发断裂检测, 因此 应该回溯一帧, 将第 $t+1$ 帧作为断裂前的最后一 帧，定义为断裂帧。 


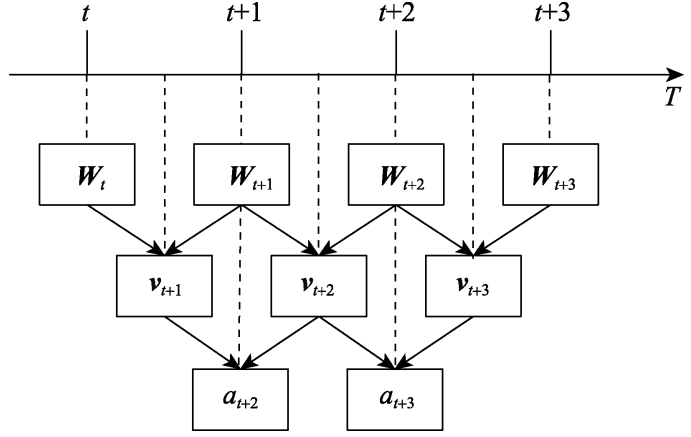

图 12 速度、加速度计算的滞后性

\section{2 护套断裂判据}

图像中存在的高斯噪声导致了计算出的三维 坐标产生随机误差，这使得即使像素点不发生移 动跟踪也会产生静态误差. 可以认为, 在护套匀速 拉伸过程中加速度 $a$ 的概率密度 $f(a)$ 服从单边正 态分布，当 $a>0$ 时有

$$
f\left(a \mid \mu, \sigma^{2}\right)=\frac{2}{\sqrt{2 \pi \sigma^{2}}} \mathrm{e}^{-\frac{(a-\mu)^{2}}{2 \sigma^{2}}}
$$

其中, $\sigma$ 为加速度序列的标准差; $\mu$ 为加速度序 列的平均值，拉伸过程中 $\mu=0$. 当护套发生断裂 时, 至少一个标记点的加速度突然增大, 则会落在 $\mu+3 \sigma$ 区间之外. 用前 25 帧的加速度数据对 $\sigma$ 进 行无偏参数估计, 后续帧的 $\sigma$ 根据缓存的加速度 序列实时更新. 为了降低误检测率：(1) 提升阈值, 将加速度阈值提升到 $a>5 \sigma$ ；(2) 发生断裂时加速 度应大于一定的常数, 本文取 $5 \mathrm{~cm} / \mathrm{s}^{2}$. 当至少一个 标记点的加速度满足上述 2 个条件时, 读取当前帧 的前一采样帧缓存的标记点距离作为断裂长度, 并作为断裂帧.

\section{3 采样粗检测与逐帧精检测}

图 13 所示为本阶段的直观示意图, 首先隔 $k$ 帧采样视频，虚线框的帧为采样跳过的帧，实线框 的帧为采样帧，可以看到，深色的帧为断裂发生前 的最后一个采样帧，第 4.2 节中将其定义为断裂帧， 算法检测出断裂的浅色帧在粗检测和精检测中都 处在断裂帧之后一帧，为了方法的稳定性，回溯 2 帧进行逐帧精检测, 直至再次检测到断裂. 一次断 裂检测的处理时间为

$$
T_{\mathrm{d}}=\left(2 k+\frac{F}{k}\right) T_{0}
$$

其中， $F$ 为视频从开始到断裂的帧数; $T_{0}$ 为每帧 平均处理时间, 可知当且仅当 $k=\sqrt{F / 2}$ 时处理时 间有最小值. 通常, $F$ 取值范围为 600 800 帧, 故 采样步长 $k$ 理论上应取 17 20, 但当采样间隔取得
过大时会引起光流跟踪误差, 经过多次测试, $k=13$ 时能兼顾速度和精度. 以此法提取的电缆断 裂帧如图 14 所示，取断裂帧前一帧的标记点间距 作为最大伸长量 $l_{m}$.

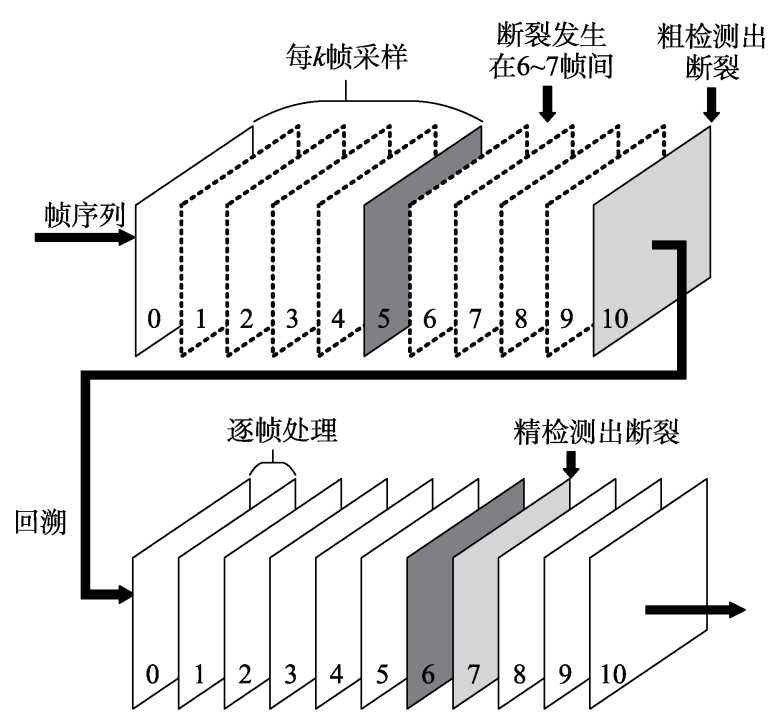

图 13 断裂检测算法示意图

电缆护套的断裂伸长率为

$$
P_{m}=\frac{l_{m}-l_{0}}{l_{0}} \times 100 \%
$$

其中, $l_{0}$ 为护套上标记距离的原始长度 $; l_{m}$ 为最 大伸长量. 通常 $P_{m}$ 大于一定阈值, 则认为该种类 的电缆护套的质量达标. 最后, 计算断裂帧精确定 位和断裂伸长率.

算法 2. 断裂帧精确定位和断裂伸长率计算.

输人. 处理过程双目视频; 左右相机的投影 矩阵 $\boldsymbol{M}_{1}, \boldsymbol{M}_{2}$ ；采样间隔 $k$.

输出. 断裂伸长率 $\rho$.

Step1. 计算首帧中标记点的初始间距 $l_{0}$, 设检测 状态标志位 $f_{d}=0$, 表示当前处于隔帧采样阶段.

Step2. 实时跟踪和三维信息计算.

repeat

if $f_{d}=0$ then

将采样间隔置为 $k$,

else

将采样间隔置为 1

end if

读取采样帧, 用金字塔 $\mathrm{L}-\mathrm{K}$ 光流法跟踪 2 个标记点.

计算标记点的像素坐标 $\boldsymbol{x}_{m}$, 基于 $\boldsymbol{M}_{1}, \boldsymbol{M}_{2}$ 参数和 $\boldsymbol{x}_{m}$ 计算三维坐标 $\boldsymbol{W}_{m}$, 进而计算速度 $\boldsymbol{v}_{m}$ 、加速度 $a_{m}$ 以 及标记点当前帧的三维间距 $l_{m}$, 全部人栈.

if 已处理的采样帧数 $n_{p}>25$ then

$$
\text { if } a_{m}>5 \sigma \text { and } a_{m}>5 \mathrm{~cm} / \mathrm{s}^{2} \text { then }
$$




$$
\text { if } f_{d}=0 \text { then }
$$

出栈 $2 k$ 组参数 $(x, W, v, a, l)$, 并将 $f_{d}=1$, 开始逐帧检测.

else

出栈上一帧的标记点间距，计算并输出断 裂伸长率 $\rho=\left(l_{m-1}-l_{0}\right) / l_{0}$.

break

end if

end if

end if

until 视频结束

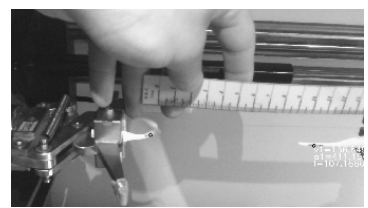

a. 提取结果 1

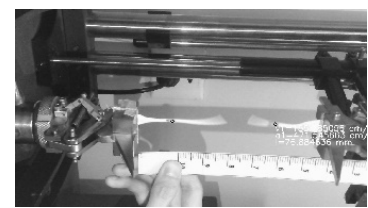

b. 提取结果 2
图 14 断裂帧提取结果

\section{5 实验结果及分析}

本文实验使用的是工业双目摄像头，单目分 辨率为 $1280 \times 720$, 传输帧率为 30 帧 $/ \mathrm{s}$, 使用 $\mathrm{C}++$ 语言进行编程, 调用了 OpenCV 库, 在 Intel(R) i5-10210U $1.60 \mathrm{GHz}$ CPU, $16 \mathrm{~GB}$ 内存的个人计算 机上实现.

本文进行了 27 组重复实验，测量距离为 $45 \sim 50 \mathrm{~cm}$. 首先用游标卡尺对护套的初始长度进 行测量作为标记的初始长度真值，再同时采用本 文算法和量尺目测法进行断裂伸长率检测，量尺 目测法的操作如图 15 所示. 采用均方根误差(root mean square error, RMSE)和均方根百分误差(root mean square percentage error, RMSPE)评价实验测 量结果, 实验测得的数据如表 1 所示. 可见, 针对 与双目相机间距在 $45 \sim 50 \mathrm{~cm}$ 范围内的待测目标, 本文对真值平均为 $18.201 \mathrm{~mm}$ 的标记点间距进行 三维测量结果均方误差为 $0.4212 \mathrm{~mm}$, 大于用最小 刻度为 $1 \mathrm{~mm}$ 的量尺测量时 $0.2342 \mathrm{~mm}$ 的误差.

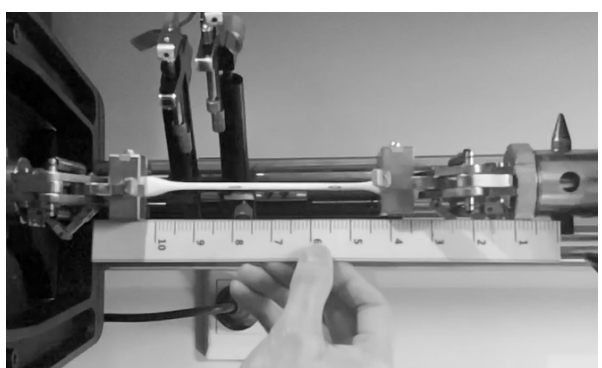

图 15 量尺目测法的测量条件
表 1 标记点初始距离双目测量误差

\begin{tabular}{ccccc}
\hline 方法 & $\begin{array}{c}\text { 测量值 } \\
\text { (平均) } / \mathrm{mm}\end{array}$ & $\begin{array}{c}\mathrm{RMSE} / \\
\mathrm{mm}\end{array}$ & $\begin{array}{c}\mathrm{RMSPE} / \\
\%\end{array}$ & $\begin{array}{c}\text { 真值 } \\
\text { (平均) } / \mathrm{mm}\end{array}$ \\
\hline 本文 & 18.3910 & 0.4212 & 2.314 & 18.201 \\
量尺 & 18.3147 & 0.2342 & 1.285 & \\
\hline
\end{tabular}

2 个标记点在拉伸过程中的三维点云如图 16 所示, 某组的加速度、速度测量结果如图 17 所示, 试样长度与拉力机拉力值的变化如图 18 所示. 可 以看出拉力发生突变的时刻则为护套发生断裂的 时刻, 此时速度和加速度都突然增大了一个数量 级以上, 标记点的间距突然增大, 而拉力因为试样 断裂突然减小. 这与物理事实相符.

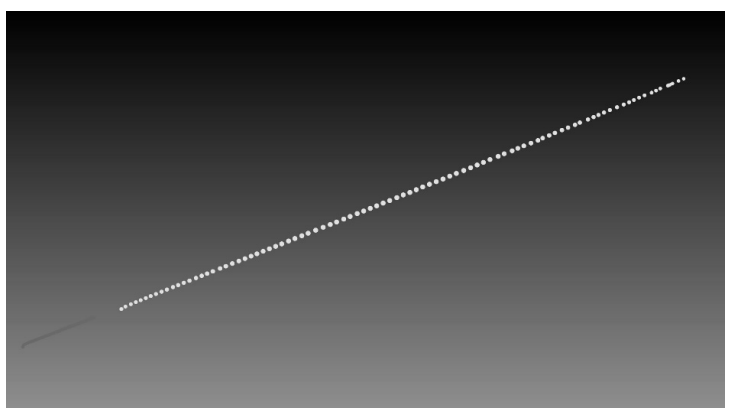

图 16 粗采样帧中标记点的三维坐标轨迹

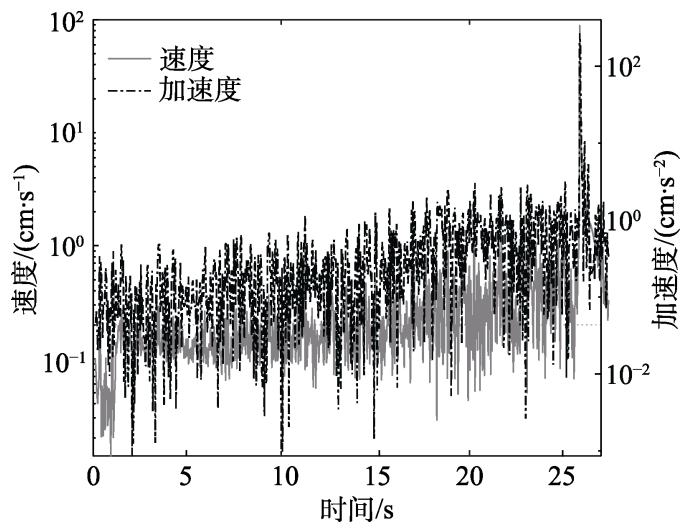

图 17 标记点速度-时间、加速度-时间图像

实际上，大部分情况下电缆断裂时的运动速 度会很快, 导致光流法产生跟踪误差, 但这样解算 出的三维坐标也会有很大偏差，导致计算出很大 的加速度，同样也能触发断裂的判定.

为了准确地获得断裂伸长率的真值, 实验采 用帧速率达 240 帧/s 的高速相机配合固定标尺进行 拍摄, 在获取的视频中逐帧读取断裂帧的拉伸长 度并计算断裂伸长率，与本文方法对比，如表 2 所 示. 可以看到量尺目测法的均方误差为 $21.84 \%$, 而本文方法的均方误差为 $7.13 \%$, 具有更高的检测 精度. 


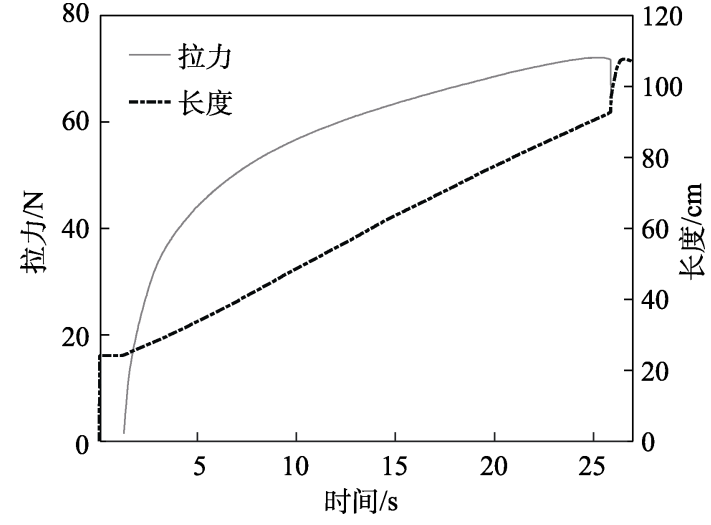

图 18 试样长度-时间、拉力-时间图像

表 2 断裂伸长率计算结果

\begin{tabular}{lcc}
\hline \multicolumn{1}{c}{ 方法 } & 断裂伸长率(平均) $/ \%$ & RMSE $/ \%$ \\
\hline 本文 & 270.88 & 7.13 \\
量尺 & 263.25 & 21.84 \\
高速相机真值 & 270.55 & \\
\hline
\end{tabular}

现有的量尺目测法的误差主要来源于: (1) 量 尺与被测试样之间难以保持平行状态; (2) 在试样 拉伸过程中测试人员很难保持正视读数, 且量尺测 量精度较低; (3) 受人的反应时间和人眼采样帧率 限制，人工读数对于断裂瞬间的定位存在误差. 这 3 方面原因导致量尺目测法误差较大，而本文方法 基本不受上述因素影响, 其测量精度高于传统方法.

在实验的测量中，双目检测装置有时会发生 轻微的抖动, 因此本文还对比了普通 L-K 光流法 和金字塔 $\mathrm{L}-\mathrm{K}$ 光流法的测量精度和跟踪效果，共 进行 27 次重复实验并取断裂长度的平均值, 如表 3 所示. 由于标记点较快的运动速度或双目检测装 置的轻微抖动, 普通 $\mathrm{L}-\mathrm{K}$ 光流法跟踪发生了 4 次失 败，只有 $85.19 \%$ 的跟踪成功率. 金字塔 L-K 光流 法成功率为 $100 \%$; 而在跟踪成功的结果中，以金 字塔 L-K 光流法的结果为真值, 与普通 L-K 光流 法断裂长度的均方误差千分比为 $1.723 \%$ ，精度基 本一致. 因此可以看出, 基于金字塔的 L-K 光流法 针对轻微的抖动具有更好的跟踪稳定性.

表 3 金字塔与普通 L-K 光流法对比

\begin{tabular}{ccrc}
\hline 方法 & $\begin{array}{c}\text { 断裂长度 } \\
\text { (平均) } / \mathrm{mm}\end{array}$ & $\begin{array}{r}\text { 跟踪成 } \\
\text { 功率 } / \%\end{array}$ & $\begin{array}{c}\text { 断裂长度均方根偏 } \\
\text { 差的千分比 } / \%\end{array}$ \\
\hline 金字塔 & 99.3482 & 100.00 & 1.723 \\
普通 & 99.3895 & 85.19 & \\
\hline
\end{tabular}

经过实验测试，本文方法的平均处理速度为 107.78 帧 $/ \mathrm{s}$, 针对 30 帧 $/ \mathrm{s}$ 的视频, 能在平均 $8.74 \mathrm{~s}$ 内处理平均时长为 $31.4 \mathrm{~s}$ 的输人视频.

\section{6 结 语}

本文提出了一种基于双目视觉的电缆护套断 裂伸长率自动检测方法. 该方法首先对试样上的 圆环标记点进行定位、跟踪和三维重建, 然后基于 重建信息获取标记点加速度, 进行断裂帧的精确 定位, 最后计算标记点间距获取断裂伸长率. 在护 套质检现场进行实验的结果表明，该方法能以平 均 107.78 帧/ $\mathrm{s}$ 的速度对输人的视频进行检测, 针 对与双目相机间距在 $45 \sim 50 \mathrm{~cm}$ 范围内的待测目标, 对真值为 $15 \sim 25 \mathrm{~mm}$ 的标记点初始间距进行三维测 量的均方误差为 $0.4212 \mathrm{~mm}$, 是真值的 $2.314 \%$, 略高 于最小刻度为 $1 \mathrm{~mm}$ 的量尺的测量误差; 以 240 帧/s 高速相机的测量结果作为断裂伸长率真值进行对 比, 本文方法测量结果的均方误差为 $7.13 \%$, 低于 传统量尺目测法的 $21.84 \%$. 该方法检测速度快， 精度也高于人工检测结果, 不仅能明显提升质检 的自动化程度, 还满足了安全生产的要求. 同时, 该技术有利于电缆行业的新品研发和缩短产品质 检验收周期, 对生产过程中的在线控制工艺、产品 质量控制均起到一定的规范作用，还能促进电缆 质检行业测量标准的优化. 该技术也可以应用到 类似的工业检测领域中.

本文实验是在较好的光照条件下进行的，标 记点追踪算法对于光照条件较差时的阴影和亮斑 的影响不具备很强的鲁棒性, 这一问题有待进一 步研究. 此外, 在逐帧检测时, 由于标记点移动速 度过慢导致真实光流较小, 信噪比较低, 因噪声产 生的静态误差相对明显, 如何抑制这种静态误差 也具有一定的研究价值.

\section{参考文献(References):}

[1] Chen Hao, Yu Shuda. A stereo camera tracking algorithm used in glass-free stereoscopic display system[J]. Journal of Computer-Aided Design \& Computer Graphics, 2017, 29(3): 436-443(in Chinese)

(陈浩, 郁树达. 一种应用于裸眼立体显示系统的双目跟踪 算法 [J]. 计算机辅助设计与图形学学报, 2017, 29(3): 436-443)

[2] Xie Zexiao, Wang Xiaodong, Gong Hanlei. Round-hole profile measurement of thin-walled parts based on binocular vision[J]. Chinese Journal of Lasers, 2019, 46(12): 179-186(in Chinese) (解则晓, 王晓东, 宫韩否. 基于双目视觉的薄壁零件圆孔 轮廓测量 $[\mathrm{J}]$. 中国激光, 2019, 46(12):179-186)

[3] Chong Aixin, Yin Hui, Liu Yanting, et al. Research on longitudinal displacement measurement method of seamless rail based on binocular vision[J]. Chinese Journal of Scientific Instru- 
ment, 2019, 40(11): 82-89(in Chinese)

(崇爱新, 尹辉, 刘艳婷, 等. 基于双目视觉的无缝线路钢轨 纵向位移测量方法研究 [J]. 仪器仪表学报, 2019, 40(11): 82-89)

[4] Li Chengmei, Bai Hongyang, Guo Hongwei, et al. Moving object detection and tracking based on improved optical flow method[J]. Chinese Journal of Scientific Instrument, 2018, 39(5): 249-256(in Chinese)

(李成美, 白宏阳, 郭宏伟, 等. 一种改进光流法的运动目标 检测及跟踪算法[J]. 仪器仪表学报, 2018, 39(5): 249-256)

[5] Lei Zhifang, Sun Ping, Dai Qing. Discussion on resolution and measuring range of typical optical flow algorithm in fringe displacement measurement[J]. Acta Optica Sinica, 2020, 40(3): 149-157(in Chinese)

(类智方, 孙平, 代晴. 典型光流算法在条纹位移测量中的 分辨力和测量范围[J]. 光学学报, 2020, 40(3): 149-157)

[6] Bouguet J Y. Pyramidal implementation of the Lucas Kanade feature tracker[M]. Santa Clara: Intel Corporation, Microprocessor Research Labs, 1999

[7] Zhang Dejia, Liang Shuang, Zhang Chen, et al. 3D tree skeleton reconstruction based on enhanced PyrLK optical flow algorithm[J]. Journal of Computer-Aided Design \& Computer Graphics, 2015, 27(7): 1247-1254(in Chinese)

(张德嘉, 梁爽, 张晨, 等. 基于增强 PyrLK 光流法的三维树 木骨架重建方法 [J]. 计算机辅助设计与图形学学报, 2015, 27(7): 1247-1254)

[8] Qi Naixin, Yang Xiaogang, Li Xiaofeng, et al. Visual odometry algorithm based on ORB features and LK optical flow[J]. Chinese Journal of Scientific Instrument, 2018, 39(12): 216-227(in Chinese)

(齐乃新, 杨小冈, 李小峰, 等. 基于 $\mathrm{ORB}$ 特征和 $\mathrm{LK}$ 光流的 视觉里程计算法[J]. 仪器仪表学报, 2018, 39(12): 216-227)

[9] Tang Yuxin, Sun Ping, Dai Qing, et al. New method for surface shape measurement based on Brox optical flow algorithm[J]. Acta Optica Sinica, 2020, 40(17): 112-122(in Chinese) (唐钰欣, 孙平, 代晴, 等. 基于 Brox 光流估计的物体面形 测量新方法[J]. 光学学报, 2020, 40(17): 112-122)
[10] Tsai D Y, Lee Y, Matsuyama E. Information entropy measure for evaluation of image quality[J]. Journal of Digital Imaging, 2008, 21(3): 338-347

[11] Hou Rui, Zhao Yue, Yu Yunyi, et al. Method for detecting the relative distortion rate of endoscope based on locating the centroid of image[J]. Optical Technique, 2020, 46(4): 438-442+ 471(in Chinese)

(侯芯, 赵越, 俞云逸, 等. 基于定位图像质心的内窥镜相对 畸变率检测方法研究 [J]. 光学技术, 2020, 46(4): 438-442+471)

[12] Bay H, Tuytelaars T, van Gool L. SURF: speeded up robust features[C] //Proceedings of the 9th European Conference on Computer Vision - Volume Part I. Heidelberg: Springer, 2006: 404-417

[13] Zhang Zhan, Yang Dongsheng. Image point feature extraction algorithm of circumferential binary descriptor[J]. Journal of Computer-Aided Design \& Computer Graphics, 2017, 29(8): 1465-1476(in Chinese)

(张展, 杨东升. 圆周二进制描述符的图像点特征提取方法 [J]. 计算机辅助设计与图形学学报, 2017, 29(8): 1465-1476)

[14] Yuan Liying, Liu Jia, Wang Feiyue. An improved algorithm of SURF image registration[J]. Journal of Detection \& Control, 2020, 42(2): 65-70+78(in Chinese)

(袁丽英, 刘佳, 王飞越. 基于 SURF 的图像配准改进算法 [J]. 探测与控制学报, 2020, 42(2): 65-70+78)

[15] Wang Xiangjun, Zhang Jilong, Yin Lei. Implementation and performance analysis of optical flow based motion estimation on FPGA[J]. Optics and Precision Engineering, 2019, 27(1): 211-220(in Chinese)

(王向军, 张继龙, 阴雷. 光流法运动估计在 FPGA 上的实现 与性能分析 [J]. 光学精密工程, 2019, 27(1): 211-220)

[16] Shi J B, Tomasi C. Good features to track[C] //Proceedings of the IEEE Conference on Computer Vision and Pattern Recognition. Los Alamitos: IEEE Computer Society Press, 1994: 593-600

[17] Liu K, Song J W, Lau D L, et al. Reconstructing 3D point clouds in real time with look-up tables for structured light scanning along both horizontal and vertical directions[J]. Optics Letters, 2019, 44(24): 6029-6032 